\title{
Função tiroideia, estado de humor e cognição no idoso
}

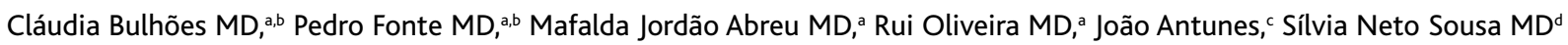

\section{RESUMO}

Objectivos: determinar a prevalência de disfunção tiroideia na população idosa e analisar a associação entre a função tiroideia e alterações cognitivas e do estado de humor.

Tipo de estudo: estudo observacional, transversal e analítico.

Local: Unidade de Saúde Familiar Ponte.

População: amostra aleatória de indivíduos com idade igual ou superior a 65 anos.

Métodos: aplicou-se um questionário relativo a características sociodemográficas e clínicas dos utentes. A função cognitiva foi avaliada pelo Mini-Mental State Examination (MMSE) e o estado de humor pela Hospital Anxiety and Depression Scale. Os níveis de hormona tiroestimulante (TSH) e levotiroxina livre foram doseados numa amostra de sangue venoso periférico. $O$ risco de apresentar disfunção cognitiva ou alteração do humor foi estimado por regressão logística não condicional, através de odds ratio (OR) e intervalos de confiança (IC 95\%). Calcularam-se médias ajustadas para a idade e escolaridade, utilizando a regressão linear múltipla, para os domínios avaliados pelo MMSE.

Resultados: avaliaram-se 263 utentes (52,5\% do género feminino), com idade média de 72,1 anos ( $\pm 5,3)$. Do total da amostra, $21,7 \%$ obtiveram uma pontuação sugestiva de demência, $12 \%$ de ansiedade e $16,8 \%$ de depressão. Quatro utentes apresentavam hipotiroidismo (1,5\%), 14 hipotiroidismo subclínico (5,3\%), 3 hipertiroidismo (1,1\%) e 2 hipertiroidismo subclínico (0,8\%). Valores mais altos de TSH demonstraram-se significativamente associados a ansiedade (OR = 1,25 IC 95\% 1,01-1,67). Não foram encontradas diferenças significativas entre o valor de TSH e disfunção cognitiva e depressão. A prevalência de demência foi significativamente superior nos doentes com depressão e/ou ansiedade.

Conclusões: a prevalência de disfunção tiroideia encontrada é semelhante à descrita na literatura, para idosos da comunidade. Verificou-se uma associação entre os valores de TSH e ansiedade. Não foi encontrada associação entre os níveis das hormonas tiroideias e a presença de disfunção cognitiva ou sintomas depressivos.

Palavras-chave: Hipotiroidismo; Hipertiroidismo; Distúrbios Cognitivos; Depressão; Ansiedade; Idoso.

\section{INTRODUÇÃO}

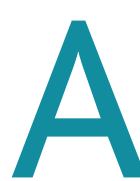
disfunção tiroideia, principalmente a subclínica, é um problema relativamente comum nos idosos. ${ }^{1,2}$ Não existe concordância entre os diversos estudos realizados até à data, no que respeita à prevalência desta condição, mas estima-se que a prevalência de hipotiroidismo, nesta população, varie entre $3,3 \%-12 \%$ e de hipertiroidismo entre $2,6 \%-5,5 \% .^{2-4}$

A disfunção tiroideia divide-se em hipertiroidismo, hipertiroidismo subclínico, hipotiroidismo e hipotiroidismo subclínico, de acordo com os níveis séricos das

a Interno(a) de Medicina Geral e Familiar na USF Ponte

${ }^{\mathrm{b}}$ Assistente Convidado(a) da Escola de Ciências da Saúde da Universidade do Minho

${ }^{c}$ Aluno do $6^{\circ}$ ano do Mestrado Integrado em Medicina da Escola de Ciências da Saúde da Universidade do Minho

${ }^{d}$ Assistente de Medicina Geral e Familiar na UCSP Cabreiros hormonas tiroestimulante (TSH), tiroxina livre (T4 livre) e triiodotironina livre (T3 livre). ${ }^{5}$ No hipertiroidismo existem níveis elevados de T4 e/ou T3 livres, com o nível de TSH diminuído e no hipotiroidismo encontra-se um nível baixo de T4 livre e um nível elevado de TSH. No que diz respeito à disfunção tiroideia subclínica, esta caracteriza-se por uma alteração do nível sérico da TSH, associada a valores séricos normais das hormonas tiroideias. O hipotiroidismo subclínico é definido por um aumento do nível sérico de TSH, com um nível sérico normal de T3 livre e T4 livre, e no hipertiroidismo subclínico há uma diminuição do nível sérico de TSH com valores séricos normais de T3 livre e T4 livre. ${ }^{2,6}$

As alterações cognitivas são também comuns nesta faixa etária, sendo reconhecidas como um importante 
problema de saúde pública. De facto, estudos revelam que aproximadamente $17 \%$ dos idosos apresentam um comprometimento cognitivo capaz de afectar as actividades de vida diária, não implicando porém uma associação ao diagnóstico formal de demência. ${ }^{7}$

Vários estudos têm verificado uma estreita associação entre as disfunções tiroideia e cognitiva, pelo que a hipótese da disfunção tiroideia poder condicionar alterações cognitivas, que surgem durante o processo de envelhecimento, tem sido investigada, com resultados contraditórios. ${ }^{1,2,7,8}$ Por outro lado, em pacientes com disfunção tiroideia, clínica ou subclínica, também são comuns as perturbações do humor, nomeadamente ansiedade e depressão, que frequentemente melhoram com o tratamento da disfunção da tiróide..$^{5,9}$ Os estudos que têm procurado demonstrar a associação entre a ansiedade/depressão e disfunção tiroideia alcançaram resultados controversos. ${ }^{9-13}$ Adicionalmente, estas perturbações de humor nos idosos são tidas como estando subdiagnosticadas e, consequentemente, subtratadas, havendo dados que apontam para prevalências de depressão entre $9 \%$ (em idosos na comunidade) e $25 \%$ (em idosos institucionalizados). ${ }^{14}$

Em Portugal não existem estudos descritos com o objectivo de avaliar a prevalência da disfunção tiroideia na população idosa da comunidade. Com base nas divergências encontradas na literatura, torna-se pertinente, no âmbito da Medicina Geral e Familiar, avaliar a prevalência da disfunção tiroideia e o seu impacto na cognição e no estado de humor do idoso. De facto, investigações nesta área poderão ter importantes implicações, quer científicas, quer na implementação de estratégias para a sensibilização dos elementos envolvidos na protecção da saúde do idoso.

Neste contexto, este trabalho tem como objectivos determinar a prevalência de disfunção tiroideia na população idosa inscrita na Unidade de Saúde Familiar (USF) Ponte e analisar a associação entre função tiroideia, alterações cognitivas e algumas alterações do estado de humor, nomeadamente ansiedade e depressão.

\section{MÉTODOS}

Realizou-se um estudo observacional, transversal e analítico, entre Maio de 2010 e Julho de 2011, na USF Ponte (Agrupamento de Centros de Saúde do Ave II,
Guimarães-Vizela). Todos os participantes foram devidamente informados dos objectivos do projecto e assinaram o respectivo consentimento informado. Esta investigação foi aprovada pela Comissão de Ética da Administração Regional de Saúde do Norte.

A população em estudo consistiu em indivíduos inscritos na USF Ponte, com idade igual ou superior a 65 anos à data de início do projecto. De forma a concretizar os objectivos pretendidos, seleccionou-se uma amostra com base na técnica de amostragem aleatória simples. Através do software SINUS (Sistema de Informação para as Unidades de Saúde), obteve-se a informação relativa à população alvo, posteriormente transferida para o software Microsoft Excel®, para aleatorização. A dimensão amostral foi calculada através do software Epi Info 6 (função STATCALC). Assumindo um intervalo de confiança de 95\%, com uma margem de erro de 5\%, obteve-se uma amostra composta por 263 indivíduos, a partir de um total de 838 utentes. Foram excluídos os utentes sem contacto telefónico disponível ou com contacto telefónico incorrecto; utentes que não atenderam o telefone três vezes em dias diferentes; utentes que revelaram incapacidade para se deslocarem à USF durante o período do estudo (por motivos de doença crónica e/ou aguda que comprometia a sua mobilidade, assim como por inexistência de meio de transporte que permitisse a sua presença durante os períodos de aplicação dos questionários e colheita de sangue); e, por fim, utentes com deficiência audiovisual ou outra (verificada no SAM ou durante o convite para participar) que não permitissem a obtenção de todos os dados necessários ao estudo. Estes utentes foram excluídos da lista previamente seleccionada, o que implicou a adição de outros tantos casos seleccionados pelo mesmo processo aleatório.

Aos utentes incluídos no estudo foi aplicado um questionário e solicitada a colheita de uma amostra de sangue venoso periférico. Adicionalmente, confirmaram-se os dados clínicos recorrendo ao Sistema de Apoio ao Médico (SAM). Realizou-se um estudo piloto, com a finalidade de verificar a aplicabilidade e exequibilidade do questionário e listar obstáculos não previstos no protocolo experimental. Neste estudo piloto foram analisados 27 indivíduos (cerca de 10\% da dimensão da amostra calculada), seleccionados de acordo com os critérios definidos, que pertenciam a 
uma população com características semelhantes. Foram elaboradas pequenas alterações ao questionário com base nos resultados obtidos.

O questionário encontrava-se dividido em 3 secções. As secções A e B foram preenchidas pelos investigadores e a secção C era de auto-preenchimento. No caso de o utente apresentar dificuldades no preenchimento desta secção, os investigadores procederam à leitura das respectivas questões. Previamente à aplicação dos questionários, os investigadores treinaram em conjunto a aplicação dos mesmos com o objectivo de uniformizar a linguagem usada na colocação das questões e de estabelecer critérios para a aceitação das respostas.

A secção A visava obter informações sociodemográficas (género, idade, escolaridade), antropométricas (peso, altura, índice de massa corporal - IMC) e clínicas (diagnóstico de disfunção tiroideia, depressão e ansiedade, com base na medicação habitual do utente). Para avaliação da função cognitiva foi aplicado o Mini-Mental State Examination (MMSE) - secção B. Trata-se da escala mais amplamente utilizada para avaliação do estado mental, nacional e internacionalmente. Inclui itens sobre orientação temporal e espacial, registo de informação, atenção e cálculo, evocação, linguagem e construção. Cada item tem uma pontuação definida e a pontuação máxima final corresponde a 30 pontos. De uma forma geral, uma pontuação igual ou inferior a 22 sugere demência. No caso de se tratar de uma pessoa analfabeta, a pontuação considerada significativa é igual ou inferior a 15. No caso de indivíduos com escolaridade superior a 11 anos, a pontuação igual ou inferior a 27 pontos é já indicativa de demência. De acordo com a escolaridade, procedeu-se à dicotomização da pontuação nas categorias com e sem demência. Esta escala encontra-se validada para a população portuguesa. $^{14}$

A secção C era constituída pela Hospital Anxiety and Depression Scale (HADS), para avaliar os níveis de ansiedade e/ou depressão dos indivíduos em estudo. Esta escala é constituída por 14 perguntas, que se devem reportar à última semana; destas, 7 permitem avaliar o domínio relativo à ansiedade (HADS-A) e as restantes 7 o domínio da depressão (HADS-B). Cada pergunta, com um score total a variar entre 0 e 3 , tem 4 hipóteses de resposta. Em cada domínio, a pontuação global varia entre 0 e 21 . Uma pontuação final igual ou superior a 11 evidencia a presença de sinais de perturbação ansiosa/depressiva clinicamente significativa. Assim, de acordo com a pontuação obtida nos dois domínios, os indivíduos foram categorizados com apresentando ou não sinais de ansiedade e depressão. Esta escala pode ser lida ao respondente e está validada para a população portuguesa. ${ }^{15,16}$ Para obtenção e confirmação dos antecedentes clínicos, de forma mais fidedigna, foi utilizado o SAM. Através do processo clínico individual, foi recolhida informação acerca do diagnóstico prévio de diabetes mellitus, hipertensão arterial (HTA), dislipidemia, antecedentes de doença cardiovascular (insuficiência cardíaca, arritmia, isquemia cerebral, fibrilhação auricular, doença cerebrovascular, isquemia com angina, isquemia sem angina, enfarte agudo do miocárdio) e história de hábitos tabágicos.

A função tiroideia foi avaliada através do doseamento de TSH e T4 livre, numa amostra de sangue venoso periférico, obtida após um período de $12 \mathrm{~h}$ de jejum. Esta colheita foi realizada na USF Ponte, aquando da aplicação do questionário, por um técnico destacado pelo laboratório de análises clínicas que colaborou no estudo (Maria de La Salete e Irmão, Lda). A selecção de apenas um laboratório prendeu-se com a necessidade de garantir a uniformidade dos procedimentose, consequentemente, dos dados obtidos.

Relativamente à análise estatística, efectuou-se uma descrição simples de todas as variáveis, com cálculo das frequências absolutas, relativas e acumuladas. Utilizaram-se os testes Qui-quadrado, one-way ANOVA, tde Student, Mann-Whitneye Kruskal-Wallis para analisar as associações entre as variáveis em estudo: género, idade, escolaridade, índice de massa corporal, hipotiroidismo, hipertiroidismo, distúrbio cognitivo, ansiedade, depressão, HTA, diabetes, dislipidemia, doença cardiovascular e tabagismo. O risco de apresentar disfunção cognitiva ou alteração do humor foi estimado por regressão logística não condicional, através de odds ratio (OR) e intervalos de confiança (IC 95\%) e ajustado para a idade, género, escolaridade, diagnóstico prévio de diabetes, HTA, dislipidemia e antecedentes de doença cardiovascular. Para análise da performance cognitiva nos diferentes domínios avaliados pelo MMSE, procedeu-se ao cálculo de médias ajustadas para a idade e escolaridade, utilizando a regressão linear múltipla. Considerou-se existir significância esta- 
tística para valores de $p<0,05$. A análise estatística foi realizada em SPSS versão 18.0 (SPSS Inc, Chicago, Illinois) e utilizou-se ainda o STATA 9.2, para o cálculo de médias ajustadas.

\section{RESULTADOS}

Da amostra inicial, foram excluídos (e repostos) 46 utentes (17,5\%): 5 por contacto telefónico não disponível, 9 por contacto telefónico incorrecto, 12 por não atenderam o telefone três vezes em dias diferentes, 17 por revelarem incapacidade para se deslocar à USF e 3 por deficiência. Foram, assim, avaliados 263 utentes, com idade média de 72 anos ( $D P 5$ anos), dos quais 138 do sexo feminino (52,5\%). Do total de utentes, 23 $(8,7 \%)$ apresentavam disfunção tiroideia: $4(1,5 \%$; IC 95\% 0,4-3,0) hipotiroidismo, 14 (5,3\%; IC 95\% 2,7-8,0) hipotiroidismo subclínico, 3 (1,1\%; IC 95\% 0-2,7) hipertiroidismo e $2(0,8 \%$; IC $95 \% 0-1,9)$ hipertiroidismo subclínico. Relativamente ao estado de humor, 30 (12\%) utentes apresentavam sintomas compatíveis com ansiedade e $42(16,8 \%)$ revelaram sintomas depressivos, salientando-se que em 13 utentes não foi possível calcular uma pontuação global por não terem respondido à totalidade das perguntas.

Quanto à análise da função cognitiva, 57 (21,7\%) utentes obtiveram pontuações no MMSE compatíveis com demência. As restantes características da amostra encontram-se descritas no quadro I.

Os utentes com demência eram significativamente mais velhos $(73,8$ anos $v s 71,7 ; p=0,014)$ e maioritariamente mulheres, embora sem significado estatístico (quadro II). Não foi encontrada qualquer associação entre HTA, diabetes, dislipidemia, doença cardiovascular, tabagismo e sinais de disfunção cognitiva (quadro II). Não se verificou, também, associação entre os níveis séricos das hormonas tiroideias e a função cognitiva (quadro II). Quanto ao estado de humor (quadro II), utentes com demência apresentaram um score superior na HADS-A e HADS-B (9,2 vs 7,6; $p=0,032 \mathrm{e}$ 8,8 vs 6,$8 ; p=0,002$, respectivamente).

As características da amostra, de acordo com o estado de humor, estão descritas no quadro III. Os utentes com sintomas de ansiedade e depressão eram na sua maioria mulheres (respectivamente, $86,7 \%$ e $71,4 \% ; p$ $<0,001$ ). Os utentes com ansiedade apresentavam um IMC superior, comparativamente aos utentes sem

\begin{tabular}{|c|c|}
\hline Características & \\
\hline Idade, anos* & $72,1(5,3)$ \\
\hline Género feminino* & $138(52,5)$ \\
\hline \multicolumn{2}{|l|}{ Escolaridade } \\
\hline Sem escolaridade ${ }^{*}$ & $61(23,2)$ \\
\hline$<4$ anos de escolaridade ${ }^{¥}$ & $79(30,0)$ \\
\hline 4 anos de escolaridade ${ }^{*}$ & $102(38,8)$ \\
\hline $5-11$ anos de escolaridade ${ }^{*}$ & $14(5,3)$ \\
\hline$\geq 12$ anos de escolaridade ${ }^{*}$ & $7(2,7)$ \\
\hline \multicolumn{2}{|l|}{ Índice de massa corporal } \\
\hline Normal ${ }^{*}$ & $62(23,6)$ \\
\hline Excesso de peso $*$ & $116(44,1)$ \\
\hline Obesidade $¥$ & $85(32,3)$ \\
\hline Hipertensão arterial $^{*}(n=245)$ & $209(85,3)$ \\
\hline Diabetes mellitus ${ }^{¥}(n=246)$ & $68(27,6)$ \\
\hline Dislipidemia ${ }^{*}(n=243) 154$ & $(63,4)$ \\
\hline Tabagismo $(n=242)$ & $8(3,3)$ \\
\hline Doença cardiovascular $(n=246)$ & $48(19,5)$ \\
\hline \multicolumn{2}{|l|}{ Função tiroideia } \\
\hline Hipotiroidismo $¥$ & $4(1,5 ;$ IC $95 \% 0,4-3,0)$ \\
\hline Hipotiroidismo subclínico ${ }^{*}$ & $14(5,3 ;$ IC $95 \% 2,7-8,0)$ \\
\hline Hipertiroidismo* & $3(1,1 ;$ IC $95 \% 0-2,7)$ \\
\hline Hipertiroidismo subclínico & $2(0,8 ;$ IC $95 \% 0-1,9)$ \\
\hline \multicolumn{2}{|l|}{ Estado de humor $(n=250)$} \\
\hline Ansiedade ${ }^{¥}$ & $30(12,0)$ \\
\hline Depressão & $42(16,8)$ \\
\hline Demência ${ }^{*}$ & $57(21,7)$ \\
\hline
\end{tabular}

alteração do humor, enquanto os utentes com depressão apresentavam um IMC inferior $(p=0,088)$. Verificou-se uma prevalência mais baixa de HTA nos utentes com sintomas de ansiedade $(p=0,035)$ e, embora sem significado estatístico, a prevalência de diabetes foi superior nos utentes deprimidos. Relativamente à função tiroideia, doentes com quadro de ansiedade obtiveram valores superiores de TSH $(p=0,078)$. Não foi encontrada qualquer relação entre os níveis de T4 livre e 


\begin{tabular}{|c|c|c|c|}
\hline \multicolumn{4}{|c|}{$\begin{array}{l}\text { QUADRO II. Características da amostra de acordo com a } \\
\text { presença de demência. }\end{array}$} \\
\hline \multirow{2}{*}{ Características } & \multicolumn{2}{|c|}{ Presença de demência } & \multirow{2}{*}{$P$} \\
\hline & Não $(n=206)$ & $\operatorname{Sim}(n=57)$ & \\
\hline Idade, anos* & $71,7(5,0)$ & $73,8(5,8)$ & $0,014^{\alpha}$ \\
\hline Género feminino & $104(50,5)$ & $34(59,6)$ & $0,220^{2}$ \\
\hline Escolaridade, anos* & $3,3(2,9)$ & $2,9(1,9)$ & $0,364^{\beta}$ \\
\hline Índice de massa corporal * & $28,4(4,4)$ & $28,4(4,3)$ & $0,965^{\beta}$ \\
\hline Hipertensão arterial ${ }^{*}$ & $164(85,9)$ & $45(83,3)$ & $0,643^{\circ}$ \\
\hline Diabetes mellitus ${ }^{*}$ & $50(26,0)$ & $18(33,3)$ & $0,290^{2}$ \\
\hline Dislipidemia ${ }^{*}$ & $120(62,8)$ & $34(65,4)$ & $0,734^{2}$ \\
\hline Tabagismo ${ }^{*}$ & $8(4,2)$ & 0 & - \\
\hline Doença cardiovascular ${ }^{*}$ & $38(19,8)$ & $10(18,5)$ & $0,835^{\Omega}$ \\
\hline Hormona tiroestimulante* & $1,9(1,1)$ & $2,0(1,3)$ & $0,900^{\beta}$ \\
\hline Tiroxina livre* & $1,3(0,8)$ & $1,2(0,2)$ & $0,753^{\beta}$ \\
\hline Ansiedade, pontuação* & $7,6(4,3)$ & $9,2(5,2)$ & $0,032^{\beta}$ \\
\hline Depressão, pontuação* & $6,8(3,9)$ & $8,8(4,3)$ & $0,002^{\beta}$ \\
\hline \multicolumn{4}{|l|}{ * média (desvio padrão) } \\
\hline \multicolumn{4}{|l|}{${ }^{\alpha}$ Teste $t$ de Student } \\
\hline $\begin{array}{l}\text { } 2 \text { Teste Qui-quadrado } \\
\beta\end{array}$ & & & \\
\hline
\end{tabular}

alterações no estado de humor.

No que diz respeito à probabilidade dos utentes apresentarem disfunção cognitiva, estimada através da regressão logística, verificou-se, após ajuste, que a idade se associou significativamente com demência $(O R=$ 1,10, IC 95\% 1,02-1,18) (quadro IV). Ter concomitantemente sintomas de ansiedade e/ou depressão também se demonstrou estar associado a uma maior probabilidade de revelar disfunção cognitiva $(O R=1,05, I C 95 \%$ 1,00-1,16; OR = 1,07, IC95\% 1,01-1,19, respectivamente). Não foi encontrada associação entre disfunção tiroideia e demência (quadro IV).

No quadro V estão representados os odds ratio brutos e ajustados para ansiedade e depressão, de acordo com as características da amostra. Após ajuste, o sexo feminino associou-se significativamente a uma maior probabilidade de apresentar ansiedade $(O R=4,45, I C$ $95 \%$ 2,00-9,87) e depressão $(O R=2,63$, IC 95\% 1,16$5,96)$. De forma semelhante, após ajuste, observou-se relação significativa entre IMC e depressão ( $O R$ $=0,90$, IC 95\% 0,82--0,98). Utentes com diabetes apresentavam, também, uma maior probabilidade de ter sintomas compatíveis com ansiedade e depressão $(O R=2,45, I C 95 \% 1,13-5,33$ e $O R=2,37, I C 95 \%$ 1,05-5,36, respectivamente). No que concerne à função tiroideia, valores mais altos de TSH estavam significativamente associados a uma maior probabilidade de apresentar ansiedade ( $O R=1,25, I C 95 \% 1,01-1,67)$, não tendo sido verificado o mesmo para o caso da depressão. Não foram encontradas associações entre as restantes patologias crónicas avaliadas e a alterações do humor em questão.

Atendendo à pontuação nos diferentes domínios específicos do MMSE, ao avaliar a sua relação com a presença de depressão, notaram-se diferenças estatisticamente significativas nos domínios «Orientação temporal» e "Atenção e cálculo». Nestes (quadro VI), as pontuações médias (ajustadas para a idade e escolaridade) foram inferiores nos indivíduos com depressão $[(3,62 v s 4,09 ; p=0,005)$ e $(2,83 v s 3,83 ; p<0,01)$, respectivamente]. Relativamente à presença de ansiedade, foi efectuada a mesma análise comparativa, não se encontrando diferenças estatisticamente significativas.

\section{DISCUSSÃO}

Neste estudo, verificou-se que $6,8 \%$ dos utentes apresentava hipotiroidismo (englobando hipotiroidismo subclínico e clínico) e 1,9\% cumpria critérios de hipertiroidismo (subclínico e clínico). A prevalência de disfunção tiroideia encontrada é semelhante à descrita na literatura para idosos da comunidade. De acordo com os resultados destes estudos, a prevalência de idosos com níveis de TSH sugestivos de hipotiroidismo varia entre $3,3 \%$ e $12 \%$ e de hipertiroidismo entre $2,6 \%$ e $5,5 \%{ }^{2-4}$ Paralelamente, ao analisar especificamente o hipotiroidismo subclínico, disfunção tiroideia mais prevalente neste estudo $(5,3 \%)$, verifica-se que esta estimativa é superior à descrita para uma população europeia com características semelhantes. De facto, segundo um estudo realizado na população inglesa, a prevalência do hipotiroidismo subclínico em indivíduos com mais de 65 anos de idade é de 2,9\%. ${ }^{5}$ 
Encontrou-se uma prevalência de disfunção cognitiva, nesta população, de $21,7 \%$, o que não difere muito do valor apontado na literatura. ${ }^{6,15}$ Por sua vez, $28,8 \%$ dos indivíduos apresentavam algum tipo de perturbação do humor. Estes resultados estão de acordo com um estudo realizado na população idosa espanhola, que tinha como principal objectivo determinar a prevalência de sintomas psiquiátricos e doenças mentais, em idosos que recorriam aos Cuidados de Saúde Primários. ${ }^{15}$ Segundo os autores, nos 293 idosos avaliados, verificou-se uma prevalência de sintomas de ansiedade e depressão de $15,7 \%$ e $14,3 \%$, respectivamente. ${ }^{15}$ É importante referir, ainda, que aquando da análise dos resultados foram detectados 10 casos em que coexistiam simultaneamente perturbação depressiva e ansiosa, os quais foram introduzidos no grupo da depressão. Apesar de se tratar de um número pequeno, não se poderá descartar completamente a hipótese desta classificação poder alterar os restantes resultados.

Não foi verificada associação entre os níveis de TSH e/ou T4 livre e a presença de disfunção cognitiva. Gussekloo Jet al, 2004, num estudo prospectivo de 599 idosos, também constataram que os níveis plasmáticos de TSH e T4 livre não estavam relacionados com incapacidade nas actividades da vida diária e alterações cognitivas. ${ }^{4}$ Roberts LM et al, em 2006, analisaram 5.865 idosos da comunidade e não encontraram associação entre a disfunção tiroideia e cognição. ${ }^{2}$ No entanto, outros estudos mostraram esta associação., ${ }^{3,6,8,16}$ As discrepâncias nos resultados poderão dever-se ao número reduzido de doentes com disfunção tiroideia encontrado em cada estudo, a diferenças nas características da amostra e nas idades analisadas, aos critérios de exclusão aplicados em cada estudo, aos diferentes valores de referência para as hormonas tiroideias e à utilização de diferentes instrumentos para avaliação da função cognitiva. Paralelamente, os valores do doseamento

\begin{tabular}{|c|c|c|}
\hline \multirow{2}{*}{ Características } & \multicolumn{2}{|c|}{ OR (IC 95\%) } \\
\hline & Brutos & Ajustados $^{\mathrm{a}}$ \\
\hline Idade, anos & $1,08(1,02-1,14)$ & $1,10(1,02-1,18)$ \\
\hline Género feminino & $1,45(0,80-2,63)$ & $1,03(0,49-2,16)$ \\
\hline Escolaridade, anos & $0,95(0,85-1,07)$ & $1,00(0,87-1,15)$ \\
\hline Índice massa corporal & $1,00(0,93-1,07)$ & $1,03(0,95-1,11)$ \\
\hline Hipertensão arterial & $0,82(0,36-1,88)$ & $0,59(0,23-1,54)$ \\
\hline Diabetes mellitus & $1,42(0,74-2,72)$ & $1,35(0,65-2,82)$ \\
\hline Dislipidemia & $1,12(0,59-2,12)$ & $1,03(0,50-2,09)$ \\
\hline Doença cardiovascular & $0,92(0,43-2,00)$ & $0,72(0,30-1,77)$ \\
\hline Hormona tiroestimulante & $1,03(0,80-1,33)$ & $1,04(0,78-1,39)$ \\
\hline Tiroxina livre & $0,89(0,48-1,65)$ & $0,96(0,45-2,08)$ \\
\hline Ansiedade, pontuação & $1,07(1,01-1,15)$ & $1,05(1,00-1,16)$ \\
\hline Depressão, pontuação & $1,13(1,05-1,21)$ & $1,07(1,01-1,19)$ \\
\hline
\end{tabular}

a $O R$ ajustados para as restantes variáveis do modelo 


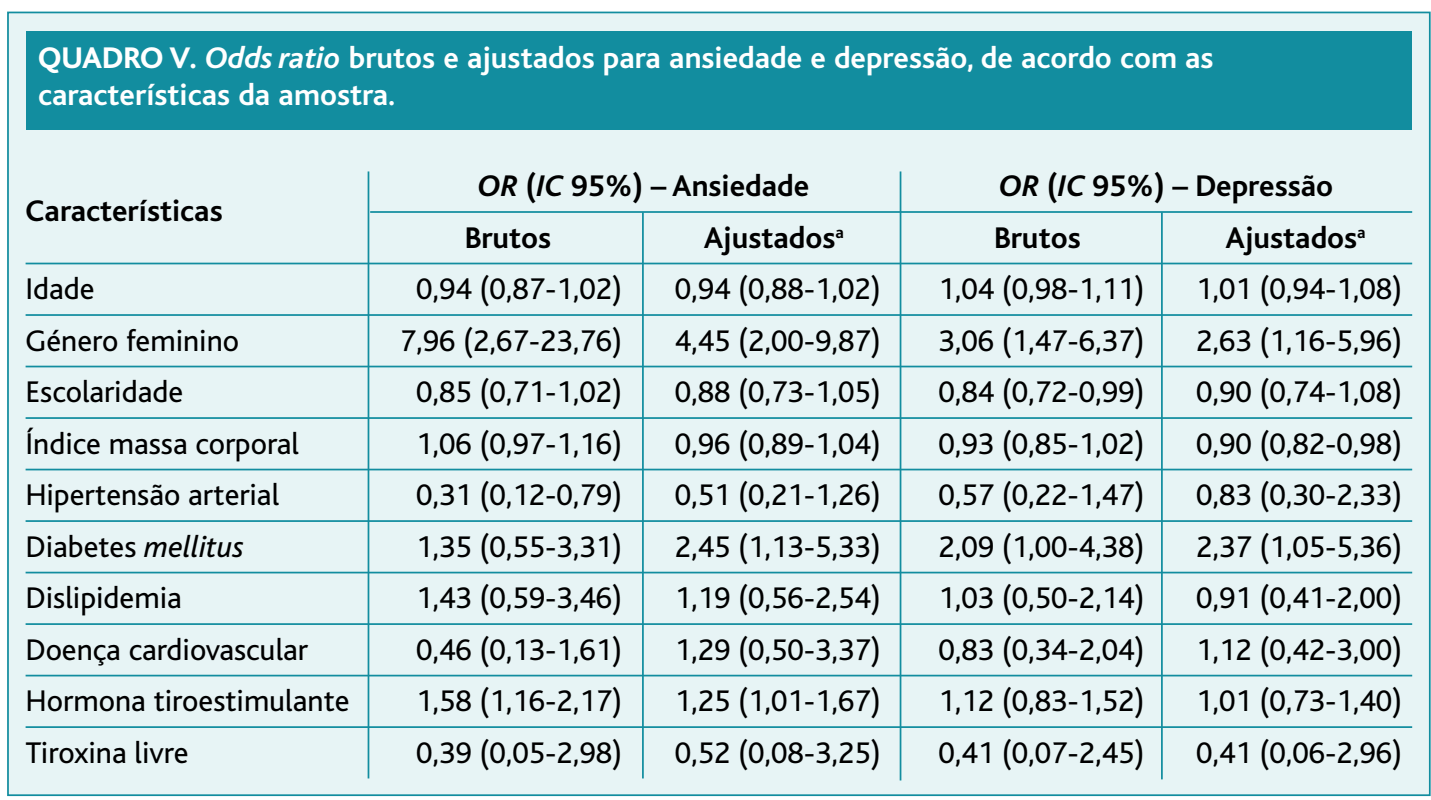

a $O R$ ajustados para as restantes variáveis do modelo

de TSH podem variar até $30 \%$, dependendo da altura do dia em que é realizada a flebotomia, assim como se os utentes estão ou não em jejum. ${ }^{17}$ Embora a maioria dos estudos que não verificou associação entre a função tiroideia e disfunção cognitiva tenha realizado as colheitas de forma aleatória, ao longo do dia, neste estu- do todas as colheitas de sangue venoso foram realizadas no período da manhã.. ${ }^{2,4}$

Neste estudo observou-se uma relação inversa entre IMC e a probabilidade de apresentar depressão. Esta observação não é apoiada pela literatura, tendo os autores constatado que estudos prévios mostravam mes-

\begin{tabular}{|c|c|c|c|c|c|c|}
\hline \multirow[t]{2}{*}{ Domínio cognitivo } & \multicolumn{2}{|c|}{$\begin{array}{c}\text { Ansiedade } \\
\text { [Média (IC 95\%)] }\end{array}$} & \multirow[t]{2}{*}{$p$} & \multicolumn{2}{|c|}{$\begin{array}{c}\text { Depressão } \\
\text { [Média (IC 95\%)] }\end{array}$} & \multirow[t]{2}{*}{$p$} \\
\hline & Não $(n=233)$ & $\operatorname{Sim}(n=30)$ & & Não $(n=221)$ & $\operatorname{Sim}(n=42)$ & \\
\hline Orientação temporal & $4,06(3,92-4,20)$ & $3,83(3,57-4,09)$ & 0,125 & $4,09(3,96-4,22)$ & 3,62 (3,32 - 3,92) & 0,005 \\
\hline Orientação espacial & $4,54(4,44-4,65)$ & $4,42(4,22-4,61)$ & 0,262 & $4,52(4,43-4,62)$ & $4,39(4,17-4,62)$ & 0,305 \\
\hline Retenção & $2,95(2,90-2,99)$ & $3,00(2,91-3,09)$ & 0,318 & $2,97(2,92-3,01)$ & $2,92(2,82-3,02)$ & 0.415 \\
\hline Atenção e Cálculo & $3,76(3,54-3,98)$ & $3,36(2,95-3,77)$ & 0,088 & $3,83(3,63-4,04)$ & $2,83(2,36-3,30)$ & $<0,001$ \\
\hline Evocação & $1,78(1,65-1,91)$ & $1,55(1,31-1,78)$ & 0,094 & $1,77(1,65-1,89)$ & $1,50(1,23-1,78)$ & 0,079 \\
\hline Linguagem/Nomeação & $2,93(2,90-2,97)$ & $2,97(2,91-3,03)$ & 0,333 & $2,94(2,91-2,97)$ & $2,94(2,87-3,01)$ & 0,973 \\
\hline Compreensão & $2,50(2,40-2,60)$ & $2,45(2,27-2,64)$ & 0,646 & $2,49(2,39-2,59)$ & $2,51(2,29-2,73)$ & 0,873 \\
\hline Leitura & $0,96(0,93-0,98)$ & $0,98(0,93-1,01)$ & 0,335 & $0,96(0,94-0,99)$ & $0,95(0,89-1,01)$ & 0,736 \\
\hline Escrita & $0,46(0,39-0,52)$ & $0,41(0,29-0,53)$ & 0,534 & $0,44(0,38-0,41)$ & $0,39(0,26-0,53)$ & 0,511 \\
\hline Visuoconstrução & $0,33(0,27-0,39)$ & $0,27(0,16-0,38)$ & 0,337 & $0,33(0,28-0,39)$ & $0,25(0,12-0,38)$ & 0,239 \\
\hline
\end{tabular}


mo o oposto. ${ }^{18}$ Nos estudos internacionais em que esta relação é directamente avaliada, constatou-se um aumento do risco de ocorrer depressão com o aumento do IMC, e que reduções dos valores de IMC se associam a uma diminuição da prevalência de sintomas depressivos. ${ }^{18}$ De notar, porém, que nenhum destes estudos envolvia apenas populações idosas, sendo esta característica diferenciadora uma possível explicação para a relação inversa observada neste trabalho. A gordura corporal é um aspecto geralmente considerado como sinal de riqueza e bem-estar, sobretudo pela população idosa portuguesa, o que se diferencia da imagem corporal que as pessoais mais jovens e com IMC superior têm de si, favorecedora do aparecimento de sintomas depressivos nos estudos citados.

Vários estudos demonstram uma associação entre disfunção tiroideia e depressão, ${ }^{9,19}$ enquanto outros, nomeadamente a presente investigação, não verificaram relação entre os níveis de TSH e/ou T4 livre e a presença de sintomas depressivos. ${ }^{2,4,20-22}$ Estes resultados inconsistentes podem dever-se a características da amostra, uma vez que esta associação é descrita maioritariamente em estudos que utilizaram amostras de base hospitalar. ${ }^{23,24}$ Para além disso, a utilização de diferentes instrumentos ou de entrevistas clínicas diagnósticas para avaliar a presença de sintomas depressivos pode, por sua vez, influenciar os resultados obtidos.

Neste estudo, verificou-se uma prevalência mais baixa de HTA nos utentes com sintomas de ansiedade. A associação entre HTA e ansiedade tem sido muito estudada, com resultados divergentes. ${ }^{25}$ Neste contexto torna-se importante referir o estudo de Davies SJ et al, de 2012, que demonstra a presença de uma associação não linear - curva em U - em que o pânico está associado a tensão arterial sistólica elevada e os sintomas de ansiedade generalizada a tensão arterial sistólica mais baixa. ${ }^{26}$ Sendo assim, fica em aberto a hipótese dos doentes estudados terem uma perturbação de ansiedade generalizada, embora a associação encontrada se possa dever apenas a uma casualidade.

Relativamente à associação entre os valores de TSH e a prevalência de ansiedade verificou-se que, independentemente do género, idade e escolaridade, os utentes com níveis mais altos de TSH tinham uma probabilidade superior de apresentar ansiedade. Esta associação ${ }^{29}$ está descrita na literatura e pensa-se que está relacionada com alterações envolvendo neurotransmissores, com aumento do número de receptores de catecolaminas em diferentes áreas do córtex cerebral, com consequentes implicações no desenvolvimento de perturbação de ansiedade. ${ }^{27-29}$ Neste sentido, Denicoffet $a l$, em 1990, demonstraram que pacientes submetidos a tiroidectomia, cujo tratamento de substituição hormonal era interrompido, desenvolviam sintomas de ansiedade.$^{30}$ Esta associação poderá revestir-se de particular importância uma vez que tem consequências na qualidade de vida dos doentes. Para além disso, o tratamento mais adequado destes doentes com quadros de ansiedade poderá conduzir à resolução da disfunção tiroideia concomitante.

Neste estudo foi, ainda, demonstrada uma possível associação entre demência e ansiedade e confirmada a associação entre demência e depressão. A literatura tem mostrado associação entre demência e depressão, mas a sua relação com ansiedade não tem sido avaliada. Desta forma, este aspecto representa um dado novo que poderá ser aprofundado em futuras investigações, para esclarecer a possível relação existente entre estas entidades clínicas. Estudos anteriores já tinham levantado a questão sobre qual dos distúrbios (disfunção cognitiva $v s$ perturbação do humor) surgiria em primeiro lugar. No entanto, está ainda por esclarecer a relação de causalidade entre estas patologias..$^{14,31}$

Quando se abordaram os diferentes domínios específicos do MMSE, para avaliar a sua associação com a prevalência de depressão e/ou ansiedade, verificaram-se diferenças estatisticamente significativas nos domínios «Orientação temporal» e "Atenção e cálculo», de acordo com a presença de depressão. Estes resultados poderão traduzir a necessidade de, na prática diária de um médico de família, se suspeitar da presença de depressão quando um idoso apresentar queixas relativas aos referidos domínios ou, alternativamente, poderão significar que, no contexto de uma depressão em idosos, aquelas serão as tarefas cognitivas mais afectadas.

Portanto, este estudo não mostrou uma associação entre disfunção tiroideia e demência, nem entre disfunção tiroideia e depressão. Em parte, tal poderá dever-se a alguns dos critérios usados na exclusão dos utentes. Isto é, os critérios «deficiência» e «impossibilidade de os utentes se deslocarem à USF» podem ter-se verificado mais frequentemente nos utentes que, 
concomitantemente, apresentassem as patologias avaliadas neste estudo - défice cognitivo, ansiedade, depressão, hipotiroidismo e hipertiroidismo.

Assim, neste estudo, os critérios de exclusão aplicados, que resultaram na exclusão de 46 unidades de observação (17,5\% da amostra), constituem uma limitação metodológica a considerar. Outra limitação importante do estudo prende-se com o cálculo da dimensão amostral, o qual, ao admitir uma margem de erro superior às prevalências mínimas dos fenómenos a estudar, resultou num subdimensionamento amostral que, por sua vez, retirou poder estatístico ao estudo. Deve considerar-se, ainda, como potencial limitação a não utilização de testes específicos para avaliação dos diferentes domínios da função cognitiva, de forma a detectar alterações ainda num estádio muito precoce. No entanto, trata-se do primeiro estudo a avaliar em Portugal a prevalência de disfunção tiroideia, tendo usado uma amostra aleatória de idosos da comunidade, com o doseamento simultâneo de TSH e T4 livre, o que permitiu diagnosticar os diferentes tipos de disfunção tiroideia existentes, e considerar outros factores cardiovasculares que poderiam ser potenciais confundidores das associações em estudo.

A disfunção tiroideia, as alterações do humor e a disfunção cognitiva são problemas comuns no idoso, por vezes interpretados no contexto do processo fisiológico do envelhecimento. No entanto, é fundamental considerar a possível associação da disfunção tiroideia com défice cognitivo e/ou alterações do humor, uma vez que o tratamento da disfunção tiroideia poderá apresentar efeitos benéficos a nível da saúde mental e física. Contudo, são necessários mais estudos, com amostras de maior dimensão e com aplicação de testes mais sensíveis para o diagnóstico de disfunção cognitiva, ainda numa fase inicial, de forma a esclarecer estas associações.

\section{AGRADECIMENTOS}

Este projecto foi premiado com a Bolsa de Investigação Clínica nas Unidades de Saúde Familiar (BICUS 2010), do grupo Tecnifar.

Os autores agradecem ao Dr. Nelson Pereira, coordenador da USF Ponte, pela sua pronta concordância na realização deste projecto na referida instituição, bem como pelos seus contínuos interesse e estímulo.

Os autores agradecem a todo o grupo de secretárias clínicas, representadas pela sua responsável (D. Fátima Azevedo), por todo o apoio prestado na recepção e organização dos utentes, aquando da colheita dos dados.

Os autores agradecem à D. Renata Ribeiro e à D. Fernanda Lourenço, pelo tempo dedicado à convocação dos doentes.

Os autores agradecem a disponibilidade e apoio do Laboratório de Análises Clínicas Maria de La Salete e Irmão, Lda, nomeadamente da Dra. Isabel Cunha e do Dr. Rocha e Costa.

\section{REFERÊNCIAS BIBLIOGRÁFICAS}

1. Benseñor IM, Lotufo PA, Menezes PR, Scazufca M. Subclinical hyperthyroidism and dementia: the Sao Paulo Ageing \& Health Study (SPAH). BMC Public Health 2010 Jun 1; 10: 298.

2. Roberts LM, Pattison H, Roalfe A, Franklyn J, Wilson S, Hobbs FD, et al. Is subclinical thyroid dysfunction in the elderly associated with depression or cognitive dysfunction? Ann Intern Med 2006 Oct 17; 145 (8): 573-81.

3. Kalmijn S, Mehta KM, Pols HA, Hofman A, Drexhage HA, Breteler MM. Subclinical hyperthyroidism and the risk of dementia. The Rotterdam study. Clin Endocrinol (Oxf) 2000 Dec; 53 (6): 733-7.

4. Gussekloo J, van Exel E, de Craen AJ, Meinders AE, Frölich M, Westendorp RG. Thyroid status, disability and cognitive function, and survival in old age. JAMA 2004 Dec 1; 292 (21): 2591-9.

5. Samuels $\mathrm{MH}$. Cognitive function in untreated hypothyroidism and hyperthyroidism. Curr Opin Endocrinol Diabetes Obes 2008 Oct; 15 (5): 429-33.

6. Ceresini G, Lauretani F, Maggio M, Ceda GP, Morganti S, Usberti E, et al. Thyroid function abnormalities and cognitive impairment in elderly people: results of the Invecchiare in Chianti study. J Am Geriatr Soc 2009 Jan; 57 (1): 89-93.

7. Parle J, Roberts L, Wilson S, Pattison H, Roalfe A, Haque MS, et al. A randomized controlled trial of the effect of thyroxine replacement on cognitive function in community-living elderly subjects with subclinical hypothyroidism: the Birmingham Elderly Thyroid study. J Clin Endocrinol Metab 2010 Aug; 95 (8): 3623-32.

8. Bégin ME, Langlois MF, Lorrain D, Cunnane SC. Thyroid Function and Cognition during Aging. Curr Gerontol Geriatr Res 2008: 474868.

9. Kritz-Silverstein D, Schultz ST, Palinska LA, Wingard DL, Barrett-Connor E. The association of thyroid stimulating hormone levels with cognitive function and depressed mood: the Rancho Bernardo study. J Nutr Health Aging 2009 Apr; 13 (4): 317-21.

10. van Boxtel MP, Menheere PP, Bekers O, Hogervorst E, Jolles J. Thyroid function, depressed mood, and cognitive performance in older individuals: the Maastricht Aging Study. Psychoneuroendocrinology 2004 Aug; 29 (7): 891-8.

11. Fatourechi V. Subclinical hypothyroidism: an update for primary care physicians. Mayo Clin Proc 2009; 84 (1): 65-71.

12. Biondi B, Cooper DS. The clinical significance of subclinical thyroid dysfunction. Endocr Rev 2008 Feb; 29 (1): 76-131.

13. Baldini IM, Vita A, Mauri MC, Amodei V, Carrisi M, Bravin S, et al. Psychopathological and cognitive features in subclinical hypothyroidism. Prog Neuropsychopharmacol Biol Psychiatry 1997 Aug; 21 (6): 925-35.

14. Wilkins $\mathrm{CH}$, Mathews J, Sheline $\mathrm{YI}$. Late life depression with cognitive impairment: evaluation and treatment. Clin Interv Aging 2009; 4: 51-7. 
15. Olivera J, Benabarre S, Lorente T, Rodríguez M, Pelegrín C, Calvo JM, et al. Prevalence of psychiatric symptoms and mental disorders detected in primary care in an elderly Spanish population. The PSICOTARD Study: preliminary findings. Int J Geriatr Psychiatry 2008 Sep; 23 (9): 915-21.

16. Hogervorst E, Huppert F, Matthews FE, Brayne C. Thyroid function and cognitive decline in the MRC Cognitive Function and Ageing Study. Psychoneuroendocrinology 2008 Aug; 33 (7): 1013-22.

17. Scobbo RR, VonDohlen TW, Hassan M, Islam S. Serum TSH variability in normal individuals: the influence of time of sample collection. WV Med J 2004 Jul-Aug; 100 (4): 138-42.

18. Fabricatore AN, Wadden TA, Higginbotham AJ, Faulconbridge LF, Nguyen $A M$, Heymsfield SB, et al. Intentional weight loss and changes in symptoms of depression: a systematic review and meta-analysis. Int J Obes (Lond) 2011 Nov; 35 (11): 1363-76.

19. Musselman DL, Nemeroff CB. Depression and endocrine disorders: focus on the thyroid and adrenal system. Br J Psychiatry Suppl 1996 Jun; (30): 123-8.

20. Engum A, Bjøro T, Mykletun A, Dahl AA. An association between depression, anxiety and thyroid function - a clinical fact or an artefact? Acta Psychiatr Scand 2002 Jul; 106 (1): 27-34.

21. Fraser SA, Kroenke K, Callahan CM, Hui SL, Williams JW Jr, Unützer J. Low yield of thyroid-stimulating hormone testing in elderly patients with depression. Gen Hosp Psychiatry 2004 Jul-Aug; 26 (4): 302-9.

22. Kim JM, Stewart R, Kim SY, Bae KY, Yang SJ, Kim SW, et al. Thyroid stimulating hormone, cognitive impairment and depression in an older korean population. Psychiatry Investig 2010 Dec; 7 (4): 264-9.

23. Chueire VB, Romaldini JH, Ward LS. Subclinical hypothyroidism increases the risk for depression in the elderly. Arch Gerontol Geriatr 2007 Jan-Feb; 44 (1): 21-8.

24. Haggerty JJ Jr, Stern RA, Mason GA, Beckwith J, Morey CE, Prange AJ Jr. Subclinical hypothyroidism: a modifiable risk factor for depression? Am J Psychiatry 1993 Mar; 150 (3): 508-10.
25. Player MS, Peterson LE. Anxiety disorders, hypertension, and cardiovascular risk: a review. Int J Psychiatry Med 2011; 41 (4): 365-77.

26. Davies SJ, Bjerkeset O, Nutt DJ, Lewis G. A U-shaped relationship between systolic blood pressure and panic symptoms: the HUNT study. Psychol Med 2012 Sep; 42 (9): 1969-76.

27. Sait Gönen M, Kisakol G, Savas Cilli A, Dikbas O, Gungor K, Inal A, et al. Assessment of anxiety in subclinical thyroid disorders. Endocr J 2004 Jun; 51 (3): 311-5.

28. Monzani F, Del Guerra P, Caraccio N, Pruneti CA, Pucci E, Luisi M, et al. Subclinical hypothyroidism: neurobehavioral features and beneficial effect of L-thyroxine treatment. Clin Investig 1993 May; 71 (5): 36771.

29. Sulser F. Serotonin-norepinephrine receptor interactions in the brain: implications for the pharmacology and pathophysiology of affective disorders. J Clin Psychiatry 1987 Mar; 48 Suppl: 12-8.

30. Denicoff KD, Joffe RT, Lakshmanan MC, Robbins J, Rubinow DR. Neuropsychiatric manifestations of altered thyroid state. Am J Psychiatry 1990 Jan; 147 (1): 94-9.

31. Rovner BW, Casten RJ, Leiby BE.Variability in depressive symptoms predicts cognitive decline in age-related macular degeneration. Am J Geriatr Psychiatry 2009 Jul; 17 (7): 574-81.

\section{ENDEREÇO PARA CORRESPONDÊNCIA}

\section{Cláudia Bulhões}

USF Ponte

Rua Reitor Joaquim Augusto Maciel Ribeiro Torres

4805-273 Ponte, Guimarães, Portugal

Email: cbulhoes@ecsaude.uminho.pt

Recebido em 18/06/2012

Aceite para publicação em 17/02/2013 


\section{ABSTRACT}

\section{THYROID DYSFUNCTION, COGNITION AND MOOD IN THE ELDERLY}

Aim: To determine the prevalence of thyroid dysfunction in the elderly and to analyze the association between thyroid function, cognition and mood.

Type of study: Observational, cross-sectional and analytical study.

Location: Ponte Family Health Unit.

Population: Random sample of individuals aged 65 and above.

Methods: A questionnaire on socio-demographic and clinical characteristics was administered. Cognitive function was assessed using the Mini-Mental State Examination (MMSE) and mood was assessed by the Hospital Anxiety and Depression Scale. Fasting blood samples were collected to measure TSH (Thyroid-stimulating Hormone) and free thyroxine levels. Odds ratios (OR) and $95 \%$ confidence interval $(95 \% \mathrm{Cl})$ were estimated using unconditional logistic regression to test the association between cognitive dysfunction and mood disorders,. Adjusted means in the domains assessed by the MMSE were calculated using multiple linear regression.

Results: We evaluated 263 individuals ( $52.5 \%$ female), with a mean age of 72.1 years $( \pm 5.3)$. In the total sample, $21.7 \%$ had a score suggesting dementia, $12 \%$ had scores suggesting anxiety and $16.8 \%$ had scores suggesting depression. Four individuals had hypothyroidism (1.5\%), 14 had subclinical hypothyroidism (5.3\%), 3 had hyperthyroidism (1.1\%) and 2 had subclinical hyperthyroidism (0.8\%). Higher TSH levels were significantly associated with anxiety $(O R=1.25 ; 95 \% \mathrm{Cl} 1.01-1.67)$. No significant differences were found between TSH levels and cognitive dysfunction and depression. The prevalence of dementia was significantly higher in patients with mood disorders.

Conclusions: The prevalence of thyroid dysfunction found in our study was comparable to published rates. There was an association found between TSH levels and anxiety. No association was found between thyroid hormone levels and the presence of cognitive impairment or depressive symptoms.

Keywords: Hypothyroidism; Hyperthyroidism; Cognitive Disorders; Depression; Anxiety; Aged. 